\title{
Repetitive Triggered Somatosensory Discrimination as a Potential Treatment for Treatment Resistant Depression and Anxiety: A Pilot Study
}

\author{
Abraham Shoshani ${ }^{1,2,3^{*}}$ \\ ${ }^{1}$ The Jerusalem Mental Health Center 'Kfar Shaul' affiliated To Hadassah Medical School Hebrew \\ University, Israel
}

${ }^{2}$ The Israeli Health Ministry, Israel

${ }^{3}$ The Helsinki Commity 'Kfar Shaul' Mental Health Center, Israel

*Corresponding author: Dr. Abraham Shoshani, The Jerusalem Mental Health Center 'Kfar Shaul' affiliated to Hadassah Medical School Hebrew University, Israel

\section{Abstract}

Background: Depression and anxiety are the most common mental disorders in Israel. Current pharmacological treatments are frequently insufficient in reducing symptoms, particularly for treatment resistant depression and anxiety. Considerable evidence has accumulated in clinical settings and research studies both with animals and humans that has implicated the involvement of the primary and secondary somatosensory and insular cortices with the regulation of emotions. Awareness deficits and irregularities of sensory experiences in the form of decreased interoceptive awareness and accuracy in both depression and anxiety have been linked to symptomology and symptom intensity which has this why this area should be a target for intervention. Nonetheless, there is a paucity of research on the effects of discriminatory somatosensory tactile stimulation on symptoms of depression and anxiety. The current pilot study aims to examine the efficacy and safety of the Repetitive Triggered Somatosensory Discrimination Device (RTSDD) in the treatment of depression and anxiety.

Methods: The present open-label pilot study included 30 outpatients diagnosed with major depressive disorder according to the DSM-V. The Hamilton Depression Rating Scale-21 (HDRS-21), the Hamilton Anxiety Rating Scale (HAM-A) as well as the Vision Analog Scale (VAS) for anxiety and depression were used for the evaluation of the severity of symptoms. A specialized device was developed called the Repetitive Triggered Somatosensory Discrimination Device (RTSDD) was utilized that provides tactile vibratory signals to the skin, foot, hand or palm at varying intensities. The device was linked to a computer program that
\end{abstract}

controlled the gradual increase of somatosensory discrimination during each of the total 8 sessions of treatment.

Results: Out of 30 participants, $21(70 \%)$ completed the trial. The mean general severity of depression (HDRS-21) was reduced from $19.48(S D=5.49)$ at baseline to 14.86 $(\mathrm{SD}=8.29)$ at week 4 and to $12.67(\mathrm{SD}=8.20)$ at week 8 . The reductions were found to be statistically significant for all measurements $(F(2,40)=9.756, P<0.001)$. The mean level of anxiety (HAM-A) was reduced from 26.09 (SD = 9.59) to $22.95(S D=12.55)$ at week 4 and to $19.19(S D$ $=11.57)$ at the final stage $(F(2,40)=5.796, P=0.006)$. The mean VAS Depression level was significantly reduced from $53.15(S D=24.52)$ at the beginning of each session to $43.84(S D=24.49)$ at the completion of each session ( $t$ $(29)=4.028, P<0.001)$. The VAS Anxiety level was also subsequently significantly reduced from 53.62 to 42.47 (t $(29)=4.537, P<0.001)$.

Limitations: The study was an open-label design with no control or placebo group and a relatively small number of participants.

Conclusions: The current study provides proof of concept for the use of the RTSDD in the treatment of depression and anxiety.

\section{Highlights}

- Awareness deficits and irregularities of sensory experiences in the form of decreased interoceptive awareness and accuracy in both depression and anxiety have been linked to symptomology and symptom intensity which is why this area should be a target for intervention.

Citation: Shoshani A (2021) Repetitive Triggered Somatosensory Discrimination as a Potential Treatment for Treatment Resistant Depression and Anxiety: A Pilot Study. Int J Depress Anxiety 4:025. doi.org/10.23937/2643-4059/1710025

Accepted: June 21, 2021: Published: June 23, 2021

Copyright: (C) 2021 Shoshani A. This is an open-access article distributed under the terms of the Creative Commons Attribution License, which permits unrestricted use, distribution, and reproduction in any medium, provided the original author and source are credited. 
- There is sufficient support within the literature for the potential use of discriminatory tactile stimulation to modulate symptoms of depression and anxiety, particularly for patients unresponsive to conventional treatments.

- The current initial pilot study shows promising results for the use of the Triggered Somatosensory Discrimination Device in the treatment of depression and anxiety.

\section{Introduction}

Depression and anxiety are the most common mental disorders in Israel with a reported combined life-time prevalence rate of $17.6 \%$ [1]. Physical, mental, and social well-being are all severely impacted by these chronic conditions and the World Health Organization has projected that they are likely to become the second leading cause of disability worldwide [2]. There is usually significant comorbidity and overlap between both major depression and anxiety [3-5]. While there are a large variety of treatments for both conditions, unfortunately first line treatments are often not effective enough in bringing about a complete relapse or in reducing symptoms to manageable levels $[3,6,7]$. While anxiety has been shown to respond better to treatment overall than depression, both disorders can often require as many as 5 stages of treatment, and sometimes even multiple simultaneous treatments for individuals who are considered to have Treatment Resistant Depression (TRD) [3,6-8]. It is therefore selfevident that there is a need for alternative treatments that can increase effectiveness in relapse prevention or symptom reduction in the contexts of monotherapy or add-on therapy for this population.

Various regions of the brain which are responsible for the receiving and processing of sensory experiences from the body including tactile attention, sensorimotor integration, and pain have also been identified in the generation, identification, and regulation of emotions including the primary and secondary somatosensory cortices, the cingulate cortices, and the anterior insula cortex [9-12]. The strong connection that has consistently been found between somatosensory experiences and emotional and cognitive processes has formed the basis of several theories of emotion [13-15]. It has therefore been suggested that interoceptive processes, or the awareness of one's inner body states and overall somatosensory experience, are an essential component of emotional regulation by modulating approach and distancing behaviors, thereby assisting to maintain or regain homeostasis (i.e, the stabilization of internal states) $[16,17]$. Indeed, considerable evidence has accumulated regarding functional as well as structural changes of the somatosensory cortex, cingulate cortices, insula hypo and hyper-connectivity, as well as pervasive disconnections between the anterior insula cortex and attentional networks in patients with depression and anxiety [17-22].
When thoughts are left undirected they may contribute adversely to the preoccupation of abnormal subjective experiences such as increased bias toward negative self-views or increased attentional biases towards threats, reinforcing inaccurate beliefs towards interoceptive signals $[17,23]$. This dysregulation of emotion can be a hallmark of depression and anxiety and may have profound effects on one's health, accordingly, it would appear that interventions that promote somatosensory integration through increased interoceptive awareness, accessibility, and tolerability could contribute significantly towards improving this [24]. It is plausible that by actively directing thoughts towards interoceptive processes the functional mechanisms for emotional regulation can be restored. This is possible since, as previously mentioned, the same path of attention and awareness of one's emotions that exists for emotional regulation seems to be intimately linked with awareness of body states [16]. Accordingly, sensorimotor activation has been one of the suggested approaches for regulating mood, particularly for depression [25].

Considerable evidence has accumulated in clinical settings and research studies both with animals and humans that has supported a bottom-up approach whereby hypo- or hyper-activation of the sensory or motor systems through external manipulations can impact depressive symptoms including visual, auditory, and olfactory stimuli and even taste [25]. There is one study that found that multisensory stimulation, which included a form of tactile stimulation among others, significantly reduced depression and anxiety among nursing home residents [26]. Several rat studies have shown reduced depression-like and anxietylike behaviors, HPA axis modulation, increased GFAP signaling, and regulation of neurotrophic factors, following tactile stimulation $[27,28]$. Other interventions aimed at promoting sensory discrimination and cortical reorganization have been shown to reduce levels of pain and discomfort in neurological pathologies, somatic tinnitus, and cerebral palsy [29-31], but there is a paucity of research on interventions that systematically utilize tactile stimulation as an adjunct treatment for depression and anxiety despite the strong theoretical foundation that exists [25].

The current pilot study seeks to provide proof of concept for a specialized device known as the Repetitive Triggered Somatosensory Discrimination Device (RTSDD) as an adjunct treatment for TRD and anxiety. Our hypothesis was that somatosensory discriminative activation through tactile vibratory stimulation would significantly reduce symptoms of depression and anxiety as an adjunct treatment for patients with TRD.

\section{Participants}

The current study was approved by the Research 
Ethics Board of the Jerusalem Mental Health Center and was conducted in accordance with the Declaration of Helsinki. All participants provided written consent to participate in the study after receiving detailed explanations of the study and its procedures, along with any potential risks.

Subjects were recruited from The Jerusalem Mental Health Center inpatient and outpatient clinics, as well as from the general community through advertisements in local magazines, newspapers, and the internet. Participants were required to be between 18-75 y.o., capable of giving consent and participating in 8 biweekly treatment sessions. They were also required to have met DSM-V criteria for major depressive disorder as assessed by a professional clinician and have persistent and significant depressive symptoms as defined by a score a 15 or higher on the Hamilton Depression Rating Scale (HAM-D). All subjects were required to have a history of failed treatment responses to at least 3 antidepressant trials, meeting the Stage III definition of TDR according to Rush [6]. This higher level of treatment resistance was used to compensate for the lack of a control group in the current study design in order that any potential symptom reduction could be more attributable to treatment effects. Subjects were included in the study regardless of whether they were currently on anti-depressant and anxiolytic treatment, but they needed to be sufficiently stable so that no changes to their treatment would be made during the study, as this would not be allowed for the entire duration of the study. Subjects were excluded if they were pregnant, had a prior medical history of skin problems, showed marked suicidality with a score of 4 or more on the Columbia Suicide Severity Rating Scale (CSSR-S), or presented with any acute physical illness that could interfere with study participation.

A total of 30 subjects were recruited, of which 16 were female $(53 \%)$ and 14 were male (47\%). 23 subjects (76.6\%) were being treated in outpatient clinics and the remaining seven subjects $(23 \%)$ were inpatients. The mean age was $50.3 \pm 11.97$ y.o. and the mean level of education was $14.76 \pm 5.03$ years of education. The overall mean duration for major depressive disorder among the participants was $12.97 \pm 11.21$

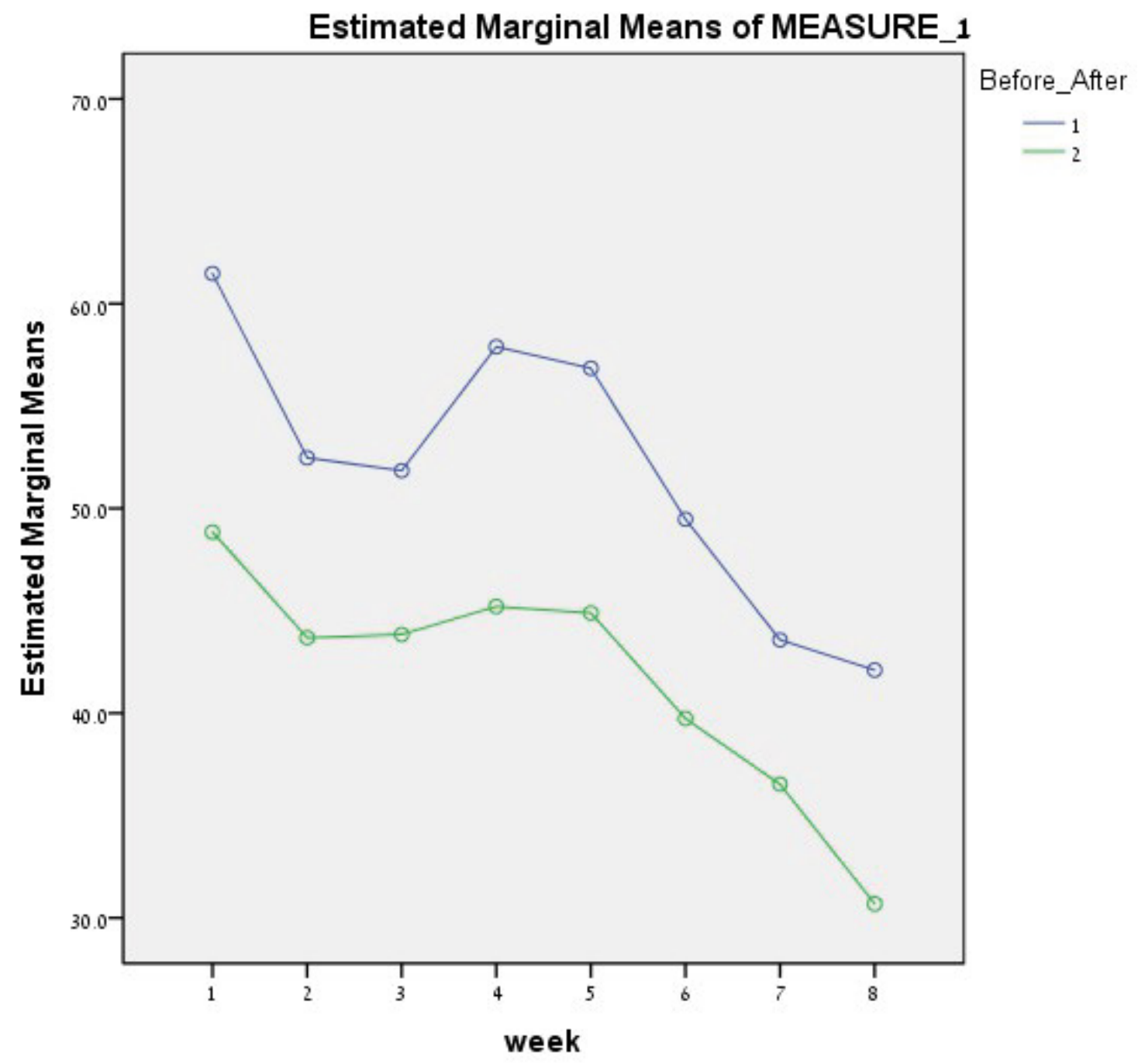

1 (Blue) = Before; 2 (Green) = After

Figure 1: VAS-Depression. 
years. 12 patients (40\%) had a history of at least one hospitalization and eight patients $(26.6 \%)$ had a history of multiple hospitalizations. Three patients had been unsuccessfully treated through ECT and one patient was unsuccessfully treated through TMS prior to entering the study. 26 patients (87\%) were being treated with SSRIs during the study and no adjustments were made until after they completed their participation. Four patients (13\%) were not taking any medication or receiving any other forms of treatment, and this remained the case until after they completed the study.

\section{Method and Device}

The repetitive triggered somatosensory discrimination device (RTSDD)

A specialized device was developed by Shoshani [32] which we will refer to as The Repetitive Triggered Somatosensory Discrimination Device (RTSDD) that provides tactile vibratory signals to the skin, foot, hand or palm at varying intensities. The current study utilized only the foot as a stimulation target. The device consists of 2 pairs of retractable moving bars which enable controlled tactile stimulation of the body (Figure 1 and Figure 2). The minimal two-point touch proximity of the bars from the foot is about two centimeters. The device is connected to an external computer program that increases and decreases the proximity of the bars to the foot from ten to two centimeters based on preprogrammed parameters. The bar's total pressure on the skin is up to 65 pascal $/ \mathrm{kg}$. Patients lie on a bed with their foot slightly off the edge of the bed in close proximity and in precise alignment with the device. An initial test is done at the beginning of each treatment session to determine the touch threshold for each patient, and adjustments are made accordingly to allow for maximum sensory discrimination. Treatments included a total of eight bi-weekly sessions of twenty minutes (ten minutes for each foot).

The study's primary endpoints of depression and anxiety were assessed by The Hamilton Depression Rating Scale-21 (HDRS-21) [33], the Hamilton Rating Scale for Anxiety (HAM-A) [34] as well as the Global Anxiety-Visual Analog Scale (GA-VAS) [22] and Depression Visual Analog Scale (D-VAS).

VAS scales for depression and anxiety were administered before and immediately after each treatment session. The HDRS-21 and HAM-A were administered at 3 different time points: 1) Baseline; 2) 4

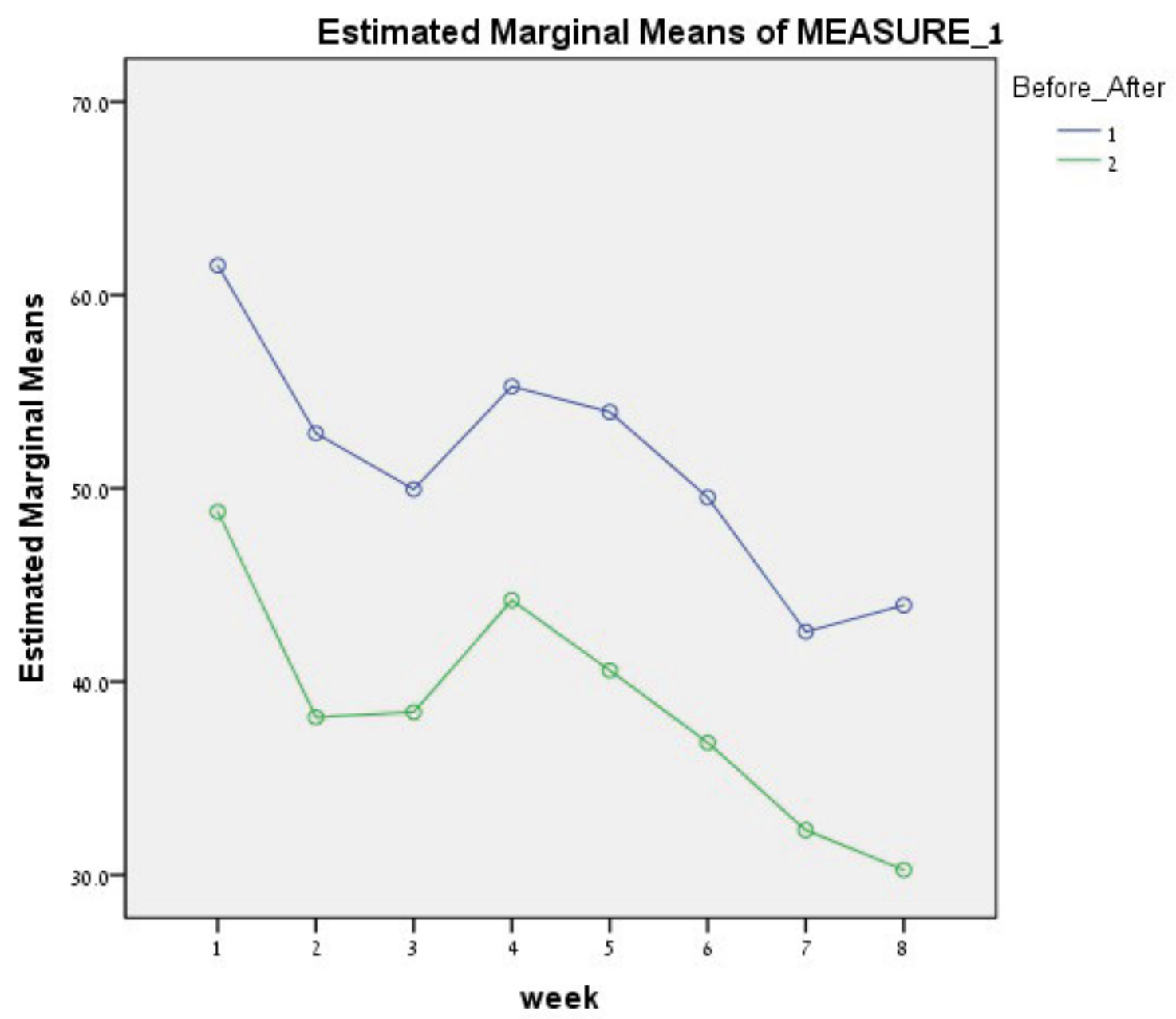

1 (Blue) $=$ Before; 2 (Green) = After

Figure 2: VAS Anxiety. 
weeks after treatment; and 3) 8 weeks after treatment.

\section{Results}

21 patients $(70 \%)$ completed the study. Out of nine patients who did not complete the study, three dropped out due to technical problems (busy schedule, transport problems, and family problems), and six patients reported no improvement and decided to withdraw at different stages. The HDRS-21 and HAM-A scores of these cases were excluded from the statistical analysis but the VAS rates of depression and anxiety before and after the treatments were included. The overall number of procedures per patient was 198. A total of 46 treatments were given to the patients who dropped out before the conclusion of the study.

No adverse events were reported during the course of the study with the exception of one female patient who reported "a general feeling of burning in the body" that began a few hours after the procedure which resolved within 48 hours and did not require any medical intervention.

The results were analyzed by ANOVA Repeated Measures. The mean general severity of depression was 19.48 (SD = 5.49) at baseline, 14.86 (SD = 8.29) at week 4 , and $12.67(S D=8.20)$ at week 8 . The differences were found to be statistically significant for all measures ( $F$ $(2,40)=9.756, \mathrm{P}<0.001)$.

The mean level of anxiety was 26.09 (SD = 9.59), 22.95 (SD = 12.55) at week 4 , and 19.19 (SD = 11.57) at the final stage of treatment. The differences were also statistically significant for all measurements $(F(2,40)=$ 5.796, $P=0.006)$

\section{The VAS measurements}

The evaluations of the levels of depression and anxiety that were obtained from the patients before and after each session were analyzed by both Paired Sample T-Tests and ANOVAs (Two Way Repeated Measures).

\section{Paired sample T-Test}

The mean D-VAS level was significantly reduced from 53.15 (SD = 24.52) pre-treatment to 43.84 (SD = 24.49) post-treatment $(\mathrm{t}(29)=4.028, \mathrm{P}<0.001)$. The GA-VAS Anxiety level was also significantly reduced from 53.62 to 42.47 ( $\mathrm{t}(29)=4.537, \mathrm{P}<0.001)$.

\section{ANOVA repeated measures two way}

Two factors were analyzed: 1) Duration, or the number of weeks (8 grades); and 2) The level of psychopathology before and after every session ( 2 grades). For D-VAS the duration was statistically significant $(F(7,126)=2.907$, $P=0.007)$. There were similar findings for levels of psychopathology before and after each session ( $F(1,18)$ $=17.134, \mathrm{P}=0.001)$. No correlation between the two factors was observed $(F(7,126)=0.725, P=0.651, N S)$. This is indicative of the stability of the improvement throughout the treatment (Figure 1).

There were similar findings for the GA-VAS scale. The duration factor was found to be significant $(F(7,26)=$ 2.692, $P=0.012$ ) and differences in psychopathology levels before and after were also highly significant ( $F$ $(1,18)=21.823, P<0.001)$. The correlation between the two factors was nonsignificant $(F(7,126)=0.374$, $P=0.916, N S)$, This, again, underlies the stability and the anxiolytic effect of the device during the 8 weeks of treatment (Figure 2).

\section{Discussion}

The results of the current pilot study provide proof of concept for the use of the RTSDD in the treatment of treatment resistant depression and anxiety. Our findings indicate significant reductions of symptoms of depression and anxiety that remained stable over the course of the treatment with minimal side-effects.

These findings provide support for the use of somatosensory stimulation and discrimination as a means of effectively modulating symptoms of depression and anxiety. This is consistent with the body of research that has linked somatosensory processing with emotional regulation [9-12], and it provides support for a bottom-up approach towards the treatment for depression and anxiety utilizing unisensory inputs of stimulation [25]. It also may shed additional light on the role of interoceptive awareness and accuracy, as well as disrupted attentional resource allocation, in depression and anxiety.

As we have mentioned previously, numerous areas of the brain, such as the primary and secondary somatosensory cortices, are involved both in processing somatosensory experiences as well as emotions [9-11], but the area that seems to be especially relevant to our findings are the insula cortices, especially the anterior insula cortex that, in conjunction with the anterior cingulate cortex, serves as a central processing hub for both somatic and emotional experiences [15,35,36], which are frequently intrinsically linked with one another and considered by some to be the neural basis for feeling states $[14,16,37]$. The insula has also been explicitly implicated as the epicenter for interoceptive awareness and human awareness in general [11,38,39], and insula hypo- and hyperconnectivity with the amygdala have been reported for both major depression and anxiety [20], and has been shown to be predictive of depression severity [21]. Both discriminative as well as affective aspects of touch, particularly stroking and vibration, have been found to be processed in the insula cortex [35]. In the current study we relied on artificial vibration as the means for tactile stimulation, due to its being highly discriminatory, particularly because it has been found to be relatively valence neutral. This specific type of non-affective stimulation has been found to have the greatest impact on the down regulation of the 
Default Mode Network (DMN) of the brain that has also been known to play a significant role in mood disorders $[35,40]$, particularly in patients who do not respond well to anti-depressant treatment [20].

Another possible explanation of our findings is that discriminatory sensory stimulation increased interoceptive accuracy, which has been found be linked to both symptoms of depression as well as anxiety [17], with research specifically indicating a negative correlation between levels of interoceptive accuracy and depression [41]. Increased interoceptive awareness and accuracy have been shown to downregulate affect-related arousal, which in turn allows for the implementation of more successful emotional regulation strategies such as cognitive reappraisal $[16,42]$. In this context it is also possible that suppression and avoidance of negative physiological and emotional experiences, a highly maladaptive response which often characterizes some of the psychopathology of patients suffering from depression and anxiety [42,43], are replaced with acceptance and non-reactiveness which leads to improved emotional regulation and health $[24,44]$. This is consistent with the model developed by Paulis [17] that emphasizes deficits in interoceptive reporting as well as disproportionate responses to negatively valenced stimuli as the fundamental dysfunctions in depression and anxiety.

Disrupted attentional resource allocation, particularly in switching between interoceptive and exteroceptive signals, has also been associated with depression and anxiety $[23,45]$. This is another way of understanding the mechanisms responsible for the effects of our intervention particularly with TRD. Enhanced somatosensory attention may produce a gating effect for external and internal stimulus. Depressed and anxious individuals are often unable to re-orient their attention away from distracting negative information towards goal-relevant information [46]. This is further enhanced through a reciprocal effect whereby low mood impairs attention and impaired attention further increases low mood [46]. Additionally, it has been shown that low mood can significantly impair individual capacity for active problem-solving and other effective strategies of emotional regulation [43,47]. Improved parameters of attention have therefore been indicated as a means towards substantial improvement in symptomology and overall quality of life [46]. This is consistent with studies that have indicated that improvements in alertness and divided attention have predicted better treatment responses in depressed individuals [48].

There are several limitations to the current study. First, our study design did not include a control group that would significantly increase the reliability of our findings. For this reason, we included individuals that were classified as having tier 3 treatment resistant depression which would make improvements in symptomology highly unlikely by time alone, but the placebo effect is still a significant confounding factor and one we could not control for. Our sample size was adequate for a pilot study but larger numbers are necessary to sufficiently support the treatment effect as well as to confirm the safety profile of the intervention. The lack of treatment blinding and drop-out rate are additional considerations. Future research will be necessary to overcome these limitations.

In summary, the findings of the current pilot study provide proof of concept for the efficacy and safety of the RTSDD in the modulation of symptoms of depression and anxiety. Treatment effects were stable over time and the device has shown to have an excellent safety profile. Large double-blind studies will be the necessary next step for establishing efficacy and safety for this device.

\section{References}

1. Levinson D, Zilber N, Lerner $Y$, Grinshpoon A, Levav I (2007) Prevalence of mood and anxiety disorders in the community: Results from the Israel national health survey. Isr J Psychiatry Relat Sci 44: 94-103.

2. Murray CJ, Lopez AD (1996) Global burden of disease and injur y series the global burden of disease. Oms 1-46.

3. Thase ME (2011) Treatment-resistant depression: Prevalence, risk factors, and treatment strategies. J Clin Psychiatry 72 : e18.

4. Ionescu DF, Niciu MJ, Mathews DC, Richards EM, Zarate CA (2013) Neurobiology of anxious depression: A review. Depress Anxiety 30: 374-385.

5. Alonso J, Lépine JP (2007) Overview of key data from the European study of the epidemiology of mental disorders (ESEMeD). J Clin Psychiatry 68: 3-9.

6. Rush JA, Trivedi MH, Wisniewski SR, Nierenberg AA, Stewart JW, et al. (2006) STAR-D (2006; AjPsych) Tiered approach for depression. Am J Psychiatry 163: 1905-1917.

7. Bystritsky A (2006) Treatment-resistant anxiety disorders. Mol Psychiatry 11: 805-814.

8. Thase ME, Rush AJ (1997) When at first you don't succeed: Sequential strategies for antidepressant nonresponders. J Clin Psychiatry 58: 23-29.

9. Kropf E, Syan SK, Minuzzi L, Frey BN (2019) From anatomy to function: The role of the somatosensory cortex in emotional regulation. Rev Bras Psiquiatr 41: 261-269.

10. Adolphs R, Damasio H, Tranel D, Cooper G, Damasio AR (2000) A role for somatosensory cortices in the visual recognition of emotion as revealed by three-dimensional lesion mapping. J Neurosci 20: 2683-2690.

11. Critchley HD, Wiens S, Rotshtein P, Öhman A, Dolan RJ (2004) Neural systems supporting interoceptive awareness. Nat Neurosci 7: 189-195.

12. Augustine JR (1996) Circuitry and functional aspects of the insular lobe in primates including humans. Brain Res Rev 22: $229-244$.

13. James W (1884) II-What is an emotion ? Mind 9: 188-205.

14. Damasio AR, Grabowski TJ, Bechara A, Damasio H, Ponto LLB, et al. (2000) Subcortical and cortical brain activity 
during the feeling of self-generated emotions. Nat Neurosci 3: 1049-1056.

15. Craig AD (2004) Human feelings: Why are some more aware than others? Trends Cogn Sci 8: 239-241.

16. Füstös J, Gramann K, Herbert BM, Pollatos O (2013) On the embodiment of emotion regulation: Interoceptive awareness facilitates reappraisal. Soc Cogn Affect Neurosci 8 : 911-917.

17. Paulus MP, Stein MB (2010) Interoception in anxiety and depression. Brain Struct Funct 214: 451-463.

18. Kang L, Zhang A, Sun N, Liu P, Yang C, et al. (2018) Functional connectivity between the thalamus and the primary somatosensory cortex in major depressive disorder: A resting-state fMRI study. BMC Psychiatry 18: 339.

19. Minuzzi L, Syan SK, Smith M, Hall A, Hall GBC, et al. (2018) Structural and functional changes in the somatosensory cortex in euthymic females with bipolar disorder. Aust N Z J Psychiatry 52: 1075-1083.

20. Goldstein-Piekarski AN, Williams LM (2019) A neural circuit-based model for depression anchored in a synthesis of insights from functional neuroimaging. Elsevier Inc.

21. DeVille DC, Kerr KL, Avery JA, Burrows K, Bodurka J, et al. (2018) The neural bases of interoceptive encoding and recall in healthy adults and adults with depression. Biol Psychiatry Cogn Neurosci Neuroimaging 3: 546-554.

22. Williams VSL, Morlock RJ, Feltner D (2010) Psychometric evaluation of a visual analog scale for the assessment of anxiety. Health Qual Life Outcomes 8: 57.

23. Hyett MP, Breakspear MJ, Friston KJ, Guo CG, Parker GB (2015) Disrupted effective connectivity of cortical systems supporting attention and interoception in melancholia. JAMA Psychiatry 72: 350-358.

24. Price CJ, Hooven C (2018) Interoceptive awareness skilIs for emotion regulation: Theory and approach of mindful awareness in body-oriented therapy (MABT). Front Psychol 9: 798.

25. Canbeyli R (2010) Sensorimotor modulation of mood and depression: An integrative review. Behav Brain Res 207: 249-264.

26. Moghaddasifar I, Fereidooni-Moghadam M, Fakharzadeh L, Haghighi-Zadeh MH (2019) Investigating the effect of multisensory stimulation on depression and anxiety of the elderly nursing home residents: A randomized controlled trial. Perspect Psychiatr Care 55: 42-47.

27. Roversi K, de David Antoniazzi CT, Milanesi LH, Rosa HZ, Kronbauer M, et al. (2019) Tactile stimulation on adulthood modifies the HPA Axis, neurotrophic factors, and GFAP signaling reverting depression-like behavior in female rats. Mol Neurobiol 56: 6239-6250.

28. Freitas D, Antoniazzi CTD, Segat HJ, Metz VG, Vey LT, et al. (2015) Neonatal tactile stimulation decreases depression-like and anxiety-like behaviors and potentiates sertraline action in young rats. Int J Dev Neurosci 47: 192-197.

29. Flor H, Denke C, Schaefer M, Grüsser S (2001) Effect of sensory discrimination training on cortical reorganisation and phantom limb pain. Lancet 357: 1763-1764.

30. Latifpour DH, Grenner J, Sjödahl C (2009) The effect of a new treatment based on somatosensory stimulation in a group of patients with somatically related tinnitus. Int Tinnitus J 15: 94-99.

31. Riquelme I, Zamorano A, Montoya P (2013) Reduction of pain sensitivity after somatosensory therapy in adults with cerebral palsy. Front Hum Neurosci 7: 1-7.

32. Shoshani A (2013) A device for the treatment of depression.

33. Hamilton Depression Rating Scale. The total hamilton depression (HAM-D) rating scale provides and indication of depression and, over time, provides a valuable guide to progress. Classification of symptoms which may be difficult to obtain can be scored.

34. Thompson E (2015) Hamilton rating scale for anxiety (HAM-A). Occup Med 65: 601.

35. Davidovic M, Starck G, Olausson H (2017) Processing of affective and emotionally neutral tactile stimuli in the insular cortex. Dev Cogn Neurosci 35: 94-103.

36. Gu X, Hof PR, Friston KJ, Fan J (2013) Anterior insular cortex and emotional awareness. J Comp Neurol 521: 33713388.

37. Damasio AR, Grabowski TJ, Bechara A (2000) Damasio 2000_PET_basic emotioin. 9: 1049-1056.

38. Craig AD (2013) Interoception: The sense of the physiological condition of the body. Curr Opin Neurobiol 13: 500-505.

39. Craig ADB (2009) How do you feel--now? The anterior insula and human awareness. Nat Rev Neurosci 10: 59-70.

40. Hamilton JP, Farmer M, Fogelman P, Gotlib IH (2015) Depressive rumination, the default-mode network, and the dark matter of clinical neuroscience. Biol Psychiatry 78: 224-230.

41. Pollatos O, Traut-Mattausch E, Schandry R (2009) Differential effects of anxiety and depression on interoceptive accuracy. Depress Anxiety 26: 167-173.

42. Aldao A, Nolen-Hoeksema S, Schweizer S (2010) Emotion-regulation strategies across psychopathology: A meta-analytic review. Clin Psychol Rev 30: 217-237.

43. Hayes SC, Wilson KG, Gifford EV, Follette VM, Strosahl K (1496) Experiential avoidance and behavioral disorders: A functional dimensional approach to diagnosis and treatment kirk strosahl group health cooperative. J Consult Olinicai Psychol 64: 1152-1168.

44. Shapero BG, Abramson LY, Alloy LB (2016) Emotional reactivity and internalizing symptoms: Moderating role of emotion regulation. Cognit Ther Res 40: 328-340.

45. Lo BCY, Allen NB (2011) Affective bias in internal attention shifting among depressed youth. Psychiatry Res 187: 125129.

46. Keller AS, Leikauf JE, Holt-Gosselin B, Staveland BR, Williams LM (2019) Paying attention to attention in depression. Transl Psychiatry 9: 1-12.

47. Lyubomirsky S, Tucker KL, Caldwell ND, Berg K (1999) Why ruminators are poor problem solvers: Clues from the phenomenology of dysphoric rumination. J Pers Soc Psychol 77: 1041-1060.

48. Mikoteit T, Hemmeter U, Eckert A, Brand S, Bischof R, et al. (2015) Improved alertness is associated with early increase in serum brain-derived neurotrophic factor and antidepressant treatment outcome in major depression. Neuropsychobiology 72 : 16-28.
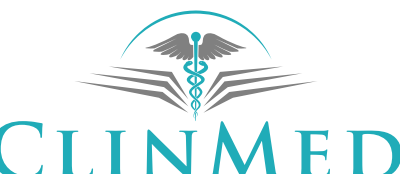

INTERNATIONAL LIBRARY 